\title{
The Dynamics of the Neutron Complexes: From Neutron Star to Black Hole
}

\author{
Yuriy Nikolaevich Zayko \\ Russian Presidential Academy of National Economy and Public Administration, Stolypin Volga Region Institute, Saratov, Russia

\section{Email address:} \\ zyrnick@rambler.ru

\section{To cite this article:} \\ Yuriy Nikolaevich Zayko. The Dynamics of the Neutron Complexes: From Neutron Star to Black Hole. International Journal of \\ Astrophysics and Space Science. Vol. 7, No. 4, 2019, pp. 36-40. doi: 10.11648/j.ijass.20190704.11
}

Received: June 27, 2019; Accepted: September 28, 2019; Published: October 11, 2019

\begin{abstract}
The mechanism of the appearance of neutron complexes, which at the final stage of their development, evolve into neutron stars, is described. It is shown that for a quantitative description it is necessary to use a generalization of the Newton-Schrödinger equations taking into account the next terms in the decomposition of explicit Dirac - Maxwell equations on $c^{-2}$. In this approximation, the problem is described by the well-known Gross-Pitaevskii equation, the numerical analysis of which is performed for the spherically symmetric case. The result depends on the value of the parameter $\alpha$ equal to the ratio of the gravitational radius of the neutron complex to twice the Compton wavelength. For small values of $\alpha<0.5$, the solutions describe a neutron star; for $\alpha>0.5$, the description corresponds to its gravitational collapse. This is consistent with the analysis of the general 3-dimensional case.
\end{abstract}

Keywords: Schrödinger-Newton Equations, Gravitational Potential, Neutron Star, Bosonic Condensate, Gross-Pitaevskii Equation

\section{Introduction}

In the previous author's work devoted to the numerical study of the Newton-Schrödinger (NS) equation, it was suggested that neutrons can combine into complexes containing a large number of particles and which determine the law of gravitational interaction at distances of the order of the size of galaxies [1]. The present work is devoted to the development of these ideas. As shown below, to solve the formulated problem, it is required to take into account in the NS equation the next-order terms in $c^{-2}$ ( $c$ is the speed of light), which leads to the Gross-Pitaevskii equation. This allows us to draw an analogy between the description of neutron stars and condensed matter physics, where this equation is used to describe the Bose condensate of elementary excitations. The basis for this analogy is the phenomenon of neutron pairing and the appearance in them of the features characteristic of Bose gases. A numerical analysis of the obtained equation is performed in the case of spherical symmetry for various values of the parameter $\alpha$, which represents the ratio of the gravitational radius of the neutron complex and it's doubled Compton wavelength. For small values of $\alpha<0.5$, the solutions describe a neutron star, for $\alpha>0.5$ - its gravitational collapse.
Thus, the present consideration throws a kind of bridge between the quantum and relativistic picture of the phenomenon. At the same time, it can be considered as a result of quantization of the Newtonian theory of gravity.

\section{Schrödinger-Newton Equations}

Let's write the time-variant of the Schrödinger-Newton equations

$$
\begin{aligned}
& i \hbar \frac{\partial \phi}{\partial t}=-\frac{\hbar^{2}}{2 m} \nabla^{2} \phi+m \Phi \phi \\
& \nabla^{2} \Phi=4 \pi G m|\phi|^{2}
\end{aligned}
$$

here $\varphi$ - complex-valued function: $\varphi=\varphi_{1}+i \varphi_{2}$, which represents the neutron wave function, $m=m_{n}$ - neutron mass, $\Phi$ - gravitational potential, $G$ - gravitational constant [2]. For the stationary spherically-symmetric variant (1) looks as follows $(\chi=r \varphi)$

$$
\begin{aligned}
& \eta_{\rho \rho}+2[v-U] \eta=0 \quad \rho^{2} U_{\rho \rho}+2 \rho U=4 \pi|\eta|^{2} \\
& \eta=\frac{\hbar}{\sqrt{G m^{3}}} \chi, U=\frac{\hbar^{2}}{\sqrt{G^{3}} m^{4}} \Phi, v=\frac{\hbar^{2}}{G^{2} m^{5}} \omega, \rho=\frac{G m^{3}}{\hbar^{2}} r
\end{aligned}
$$


here $\phi(r, t)=\phi(r) \exp (-i \varepsilon t / \hbar), \omega=\varepsilon-m c^{2}$.

Let's introduce values $N$ and $\langle R>$, which have a sense of particles' number and an average size (radius) of the neutron complex

$$
\begin{aligned}
& N=\int|\phi|^{2} d V, \\
& <R>=\int r|\phi|^{2} d V
\end{aligned}
$$

$d V$ - is an elementary $3 \mathrm{~d}$-volume, integration is spread on all space. Using (1) we receive the equations for $N$ and $<R>$

$$
\begin{aligned}
& \frac{\partial N}{\partial t}=-\int \operatorname{div} I \cdot d V \\
& \frac{\partial<R>}{\partial t}=-\int \operatorname{div}(r I) \cdot d V+\int I \cdot d V
\end{aligned}
$$

here $I=\frac{\hbar}{m}\left(\phi_{2 r} \phi_{1}-\phi_{1 r} \phi_{2}\right)$ - is a radial flux of particles. Since, as shown below, the time scale for equations (1), determined by the value of the natural unit of time, exceeds the age of the Universe, for times comparable to the time of galaxy formation, one can use the solution of the stationary system (2) to calculate the flux. This follows from the fact that the natural unit of time for the time-variant of the NewtonSchrödinger equations (1) is the quantity $2 \hbar^{3}\left(G^{2} m_{n}^{5}\right)^{-1}$, which is about $4,5 \cdot 10^{47}$ years for one neutron but greatly decreases with increasing mass of the complex.

Figures 1 and 2 show the behavior of the $J=\hbar^{7} G^{-4} m_{n}{ }^{-11} I$, где $J=\left(\eta_{1} \eta_{2 \rho}-\eta_{2} \eta_{1 \rho}\right) / \rho^{2}$.

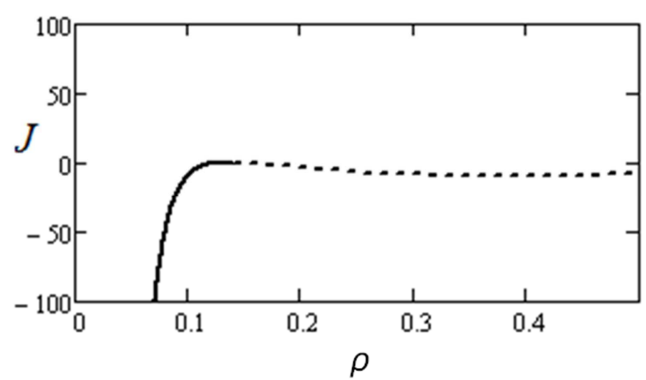

Figure 1. Dimensionless flux $J(\rho)$. The calculations were performed with the help of (2). The solid line shows the flux contributing to the formation of the neutron complex i.e. for $\rho<a$, dotted line corresponds to the contribution to the flux from other regions. The boundary conditions are set at the boundary of the stationary neutron complex, $\rho=a=0.14: \eta_{1}=-\eta_{2}=0.01, \eta_{1}^{\prime}=-\eta_{2}^{\prime}=1$, $U=0, U^{\prime}=-70$.

The first equation (4) is an integral analog of the continuity equation, which is easy to verify by representing $N=\int n d V$, where $n-$ is a particle number density and applying the Gauss theorem [3].

For the practical use of equations (4), it is necessary to determine the limits within which integration over $r$ is made. In the first integral, the upper limit of integration over $r$ can be arbitrary, $0<r<R$ then $N(r)$ will determine particles' number in the sphere of radius $r$. In the second integral, the condition is imposed on the upper limit of integration $0<\langle R>$ $<R$. In addition, from Figure 1 it can be seen that the main contribution to the particle flux is made from the distances $r$ $<a$, therefore, it is natural to impose one more condition: $R$ $<a$. In the case of spherical symmetry considered, equations (4) take the form

$$
\begin{aligned}
& \frac{\partial N(R)}{\partial t}=-4 \pi R^{2} I(R) \\
& \frac{\partial<R>}{\partial t}=R \frac{\partial N(R)}{\partial t}-\int_{0}^{R} \frac{\partial N(r)}{\partial t} d r
\end{aligned}
$$

From the presented calculations it can be seen that for the case of the corresponding Figure $1 N_{t}(R)>0$, and $\langle R\rangle_{t}>0$ i.e. the average size of the neutron complex and the number of particles in it increase in time, while for the case of the corresponding Figure 2 situation is the exact opposite. This behavior does not correspond to the concept of "complex". Therefore, research should involve additional factors.

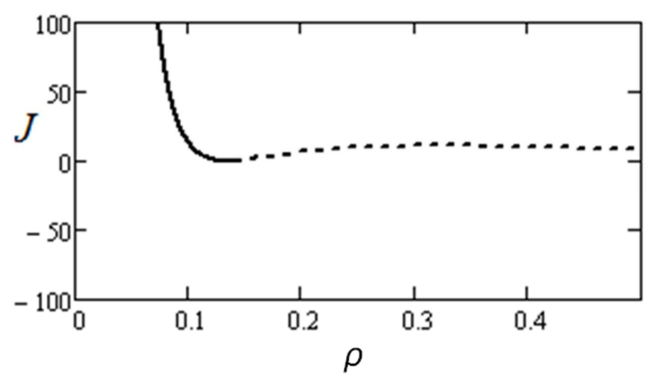

Figure 2. The same as in Figure 1 for the boundary conditions: $\eta_{1}=\eta_{2}=$ $0.01, \eta_{1}^{\prime}=\eta_{2}^{\prime}=1, U=0, U^{\prime}=-70$.

\section{The Study of the Schrödinger-Newton Equation in the Next Order in $c^{-2}$}

For this, we take into account in equations (1) the first non-vanishing terms in the decomposition of the exact DiracMaxwell equations in powers of $c^{-2}$ under the assumption of spherical symmetry [4].

$$
\begin{aligned}
& i \hbar \frac{\partial \phi}{\partial t}=\left[m c^{2}-\frac{\hbar^{2}}{2 m} \nabla^{2}+m \sqrt{G} \Phi+\frac{m \sqrt{G} \hbar^{2}}{8 m c^{2}} \Delta \Phi\right] \phi \\
& \Delta \Phi-\frac{1}{c^{2}} \frac{\partial^{2} \Phi}{\partial^{2} t}=4 \pi m \sqrt{G}|\phi|^{2}
\end{aligned}
$$

After substituting $\Delta \Phi$ from the second equation (6) ${ }^{1}$ into the first, we obtain one equation.

$$
i \hbar \frac{\partial \phi}{\partial t}=\left[m c^{2}-\frac{\hbar^{2}}{2 m} \nabla^{2}+m \sqrt{G} \Phi+\frac{\pi \sqrt{G} \hbar^{2}}{2 c^{2}}|\phi|^{2}\right] \phi
$$

In this case, the term of the second equation in (6) containing the time derivative and describing the delay can be omitted, since its influence will affect in the next order on $c^{-2}$. Presenting further $\varphi(r, t)=R(r, t) Y(\theta, \psi)$, where $Y(\theta, \psi)-$ normalized spherical harmonics, and $R(r, t)=\chi(r, t) / r$ we write the equation (7) in dimensionless variables $\rho$ (2) and $\tau=$ $G^{2} m^{5}\left(2 \hbar^{3}\right)^{-1} t$. Also, we restrict ourselves to considering small

1 Equations (6) are obtained from similar electrodynamic equations [4] by replacing the electric charge $e$ with a "gravitational charge" $m G^{1 / 2}[1]$, and not $m G$ as in [2].This leads to the replacing $\Phi$ to $G^{1 / 2} \Phi$ and will not affect the calculations. 
$\rho$, where the last term in square brackets on the right-hand side of the equation (7) behaves as $\sim \rho^{-2}$ and will prevail. Recall that the potential of the gravitational field at small distances $\Phi \sim U \sim \rho^{-1}$ and in our consideration may be omitted [1]. Then, the equation (7) takes the form

$$
\begin{aligned}
& i \frac{\partial \eta}{\partial \tau}=-\frac{\partial^{2} \eta}{\partial^{2} \rho}+\frac{\alpha^{2}}{8 \rho^{2}}|\eta|^{2} \eta \\
& \alpha=\frac{G m^{2}}{c \hbar}
\end{aligned}
$$

Dimensionless value $\alpha \approx 5,85 \cdot 10^{-39}$ is a gravitational analog of the fine structure constant in quantum electrodynamics. Its smallness leads to the fact that the influence of the last term in (8) will affect at very small distances much less the size of the Compton neutron wavelength $\lambda_{\mathrm{n}}=2 \pi \hbar\left(m_{n} c\right)^{-1}$.

However, if we apply equation (8) not to a single neutron, but a neutron complex containing a large number of particles, providing $\alpha \sim 1$, then the situation will change. The number of particles $N$ required for this is estimated as $N=\frac{\sqrt{c \hbar / G}}{m}=\frac{M_{P}}{m} \sim 10^{19}, M_{P}-$ Plank mass.

The solution of equation (8) has the form [5]

$$
\eta(\rho, \tau)= \pm u(\rho) \exp \left[i C_{1} \tau+i \vartheta(\rho)\right]
$$

where $C_{1^{-}}$is a constant, and the functions $u(\rho)$ and $\vartheta(\rho)$ obey the system of ordinary differential equations

$$
\begin{aligned}
& 2 u_{\rho}^{\prime} \vartheta_{\rho}^{\prime}+u \vartheta_{\rho \rho}^{\prime \prime}=0 \\
& u_{\rho \rho}^{\prime \prime}-C_{1} u-u\left(\vartheta_{\rho}^{\prime}\right)^{2}-\frac{\alpha^{2}}{8 \rho^{2}} u^{3}=0
\end{aligned}
$$

The first equation is integrated, which allows us to express $\vartheta^{\prime}=C_{2} / u^{2}, C_{2}-$ is a constant. Substituting this into (10), we obtain the equation for $u(\rho)$

$$
\begin{aligned}
& u^{\prime \prime}=K_{\alpha}(u, \rho) \\
& K_{\alpha}(u, \rho)=C_{1} u+\frac{C_{2}}{u^{3}}+\frac{\alpha^{2}}{8 \rho^{2}} u^{3}
\end{aligned}
$$

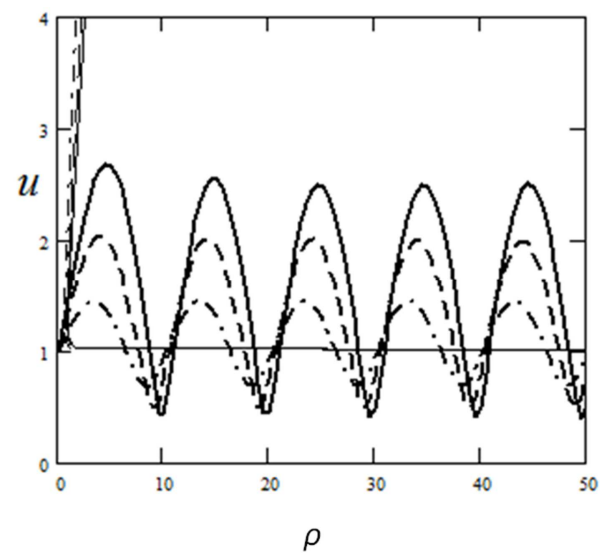

Figure 3. The results of a numerical study of equation (11). Thin lines solutions of the equation $K_{\alpha}=0$, thick lines - solutions of the equation (11) for $u(\rho)$ with boundary conditions $u(0.1)=1, u^{\prime}(0.1)=-0.03 ; C_{1}=-0.1, C_{2}$ $=0.1$. Solid lines: $\alpha=0.59$, dashed: $\alpha=0.52$, dash-dotted: $\alpha=0.4$
(We redefined the constant $C_{2}$ ). The qualitative behavior of solutions of (11) is easy to understand if we plot the curves $K_{\alpha}(u, \rho)=0$ on $u, \rho$ plane (see Figure 3). In areas adjacent to the coordinate axes $K_{\alpha}>0$ and $u^{\prime \prime}>0$, on the rest of the plane where $K_{\alpha}<0 u^{\prime \prime}<0$. This is confirmed by numerical calculation (see Figures 3, 4)

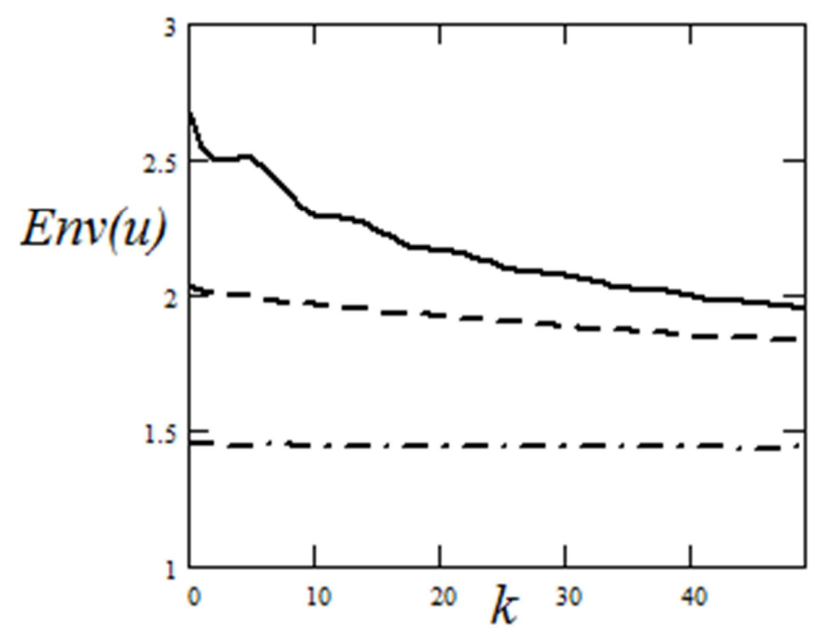

Figure 4. The envelope of solutions of equation (11), the format of the curves is the same as in Figure 3; $k$ is the oscillation number.

\section{Discussion}

A numerical study of the Schrödinger-Newton equations (1) was carried out in many works [2, 6-8]. It was shown that an initial state in the form of a Gaussian packet experiences dispersion spreading, partially restrained by the gravitational attraction of particles [2]. The results of this work are difficult to compare with that results due to the difference in the goals and methods of research.

Of interest is the relation of the results obtained to the problem of the formation of neutron stars. To do this, we make some estimates that shed light on the dynamics of the formation of neutron complexes. An increase in the number of neutrons $N$ in the complex leads to a decrease in its energy due to gravitational attraction by $G m_{n}{ }^{2} N^{2} / r, m_{n}$ - is a neutron mass, $r-$ is an average size of a complex. This process continues until the loss in energy exceeds the increase in the neutrons rest energy $\mathrm{Nm}_{n} \mathrm{c}^{2}$ and stops when a critical number of neutrons is reached $N_{\mathrm{cr}}=r c^{2}\left(G m_{n}\right)^{-1}$, what corresponds to the critical neutron density $n_{c r}=N_{c r}\left(4 \pi r^{3} / 3\right)^{-1} \sim 0,2 \cdot 10^{54} / r^{2} M^{3}$. This value is highly dependent on $r$ : if in the boundary of a galaxy $\left(r \sim 10^{21} M\right)$ it is of the order $0,2 \cdot 10^{12} M^{3}$, then on the distance of the order of neutron star $\sim 10^{4} M$ it is equal $0,2 \cdot 10^{46} M^{3}$, which coincides in order of magnitude with the real value obtained by another way [9]. Thus, the neutron complex at the final stage of its formation is a neutron star. However, it follows from the section 1 that the SchrödingerNewton equations do not make it possible to describe this process in dynamics. This also follows from the estimates given above, using the speed of light $c$, which is absent in the Schrödinger-Newton equations. From this it can be concluded that to describe neutron stars in the early stages of 
formation from neutron complexes, it is necessary to use exact equations or, at least, their expansion in powers $c^{-2}$ [4].

In this approximation, as shown above, the system of Dirac - Maxwell equations reduces to a single equation resembling the nonlinear Schrödinger equation. It is natural, therefore, to expect that additional nonlinearity will counteract the dispersion spreading of the packet noted in [2]. This is confirmed by numerical calculations. From the Figures 3 and 4 it is seen that with increasing $\alpha$, the neutron concentration increases with approaching $\rho=0$.

The qualitative behavior of the solutions of equation (11) can be explained as follows. For small $\alpha$, the nonlinear term in the equation can be neglected and its solution can be obtained by quadrature, which leads to a solution periodic in $\rho$ with period $\Delta \rho=\pi\left(-C_{1}\right)^{-1 / 2}$. With an increase in the number of neutrons in the complex, $\alpha$ grows and reaches a value of the order of unity for $N \sim 3,27 \cdot 10^{19}$. Interestingly, this value coincides in order of magnitude with the ratio of the size of a neutron star $\left(\sim 10^{4} \mathrm{M}\right)$ to the Compton neutron wavelength $(\sim$ $\left.10^{-15} \mathrm{M}\right)$.

From the other point of view $\alpha=r_{g} / 2 \lambda_{K}$, где $\lambda_{K}=\hbar / m c$ and $r_{g}=2 \mathrm{Gm} / \mathrm{c}^{2}-$ are Compton wavelength and gravitational radius of the neutron complex. Thus, the present consideration throws a kind of bridge between the quantum and relativistic picture of the phenomenon. At the same time, it can be considered as a result of quantization of the Newtonian theory of gravity.

A change of the parameter $\alpha$ from values less than 0.5 to values greater than 0.5 describes the transition of the neutron complex from the state of a neutron star to the state of a collapsing quantum object. Therefore, the solid curve in Figure 4 for which $\alpha=0.59$ refers to the collapsed neutron complex, and equation (8) for $\alpha>0.5$ represents the Schrödinger equation for a black hole.

From a quantum mechanical point of view, we are dealing with a macroscopic quantum phenomenon - the formation of the Bose condensate of paired neutrons, described by a single wave function $\varphi(r, t)[10]$.

Consider this issue in more detail. Equation (7) can be reduced to the form

$$
\begin{aligned}
& -i \psi_{\tau}-\nabla_{\rho}^{2} \psi+\pi \alpha^{2}|\psi|^{2} \psi=0 \\
& \psi=\left(\frac{\hbar}{\sqrt{G m^{3}}}\right)^{3} \phi
\end{aligned}
$$

which coincides with the well-known Gross-Pitaevskii equation used in the description of the Bose -Einstein condensate $[10]^{2}$. As stated in some works, for it there are initial conditions such that a solution cannot exist for all $\tau[11$, 12]. Similar phenomena are known in plasma physics and nonlinear optics, leading in the two-dimensional case to the collapse or self-focusing of cylindrically symmetric rays. These phenomena were observed in numerical calculations of nonlinear Schrödinger equations [13-15].

The Gross-Pitaevskii equation over the years and until recently has been used in the study of astrophysical phenomena, including describing the formation of structures of various scales - from stellar to galactic [16-20].

\section{Conclusion}

Using the Newton-Schrödinger equations, the formation of neutron complexes, including a large number of neutrons and having finite macroscopic dimensions, is studied. It is shown that neutron complexes at the final stage of their development are neutron stars. For a complete study of the dynamics of neutron complexes formation, generalizations of the Schrödinger-Newton equations should be used, taking into account the next terms in the decomposition of the explicit Maxwell-Dirac equations [4]. In this way, the GrossPitaevskii equation was obtained and its numerical analysis was performed in the case of spherical symmetry.

This work is a continuation of [1], therefore, it will be appropriate to summarize the general results. Although the Schrödinger-Newton equations as applied to cosmological problems are sometimes considered a "toy model", nevertheless, we point out some consequences of their use.

1. On a small cosmological scale, i.e. at Galactic distances, it is confirmed the Newtonian law of gravity, i.e. the attraction of massive particles of the environment to a given particle (neutron). An effective gravitational charge due to anti-screening increases. At super-galactic distances, the nature of the gravitational interaction of a neutron with its environment is repulsive. The physical cause of both phenomena is the polarization of the vacuum by the gravitational field of the neutron [1].

2. These features of the solutions of the NewtonSchrödinger equations do not contradict (at least) the observed phenomena, for the explanation of which modern science introduces the concepts of "dark matter" and "dark energy".

Finally, we note that the idea of neutron complexes arose from the desire to give a justification, in the form of some physical mechanism, the boundary conditions under which the Schrödinger-Newton equations were solved [1].

\section{References}

[1] Yu. N. Zayko, Calculation of the Effective Gravitational Charge using the Newton-Schrödinger Equations, International Journal of Scientific and Innovative Mathematical Research (IJSIMR) V. 7, № 6, 2019, PP 1722.

[2] R. Harrison, I. Moroz and K. P. Tod, A numerical study of the Schrődinger -Newton equations, Nonlinearity 16, (2003), pp. 101-122.

[3] L. D. Landau, E. M. Lifshitz (1977). Quantum Mechanics: Non-Relativistic Theory. Vol. 3 (3rd ed.), Pergamon Press.

[4] V. B. Berestetskii, E. M. Lifshitz, L. P. Pitaevskii (1971). Relativistic Quantum Theory. Vol. 4 (1st ed.), Pergamon Press. 
[5] A. D. Polyanin, V. F. Zaitsev. Handbook of Nonlinear Partial Differential Equations, (Handbooks of Mathematical Equations), 2nd Edition, Chapman and Hall/CRC, 2011.

[6] R. Penrose, On gravity's role in quantum state reduction, Gen. Rel. Grav. 28 (1996) pp. 581-600.

[7] R. Penrose, Quantum computation, entanglement and state reduction, Phil. Trans. R. Soc. (London) A 356 (1998) 1927.

[8] I. M. Moroz, R. Penrose, and P. Tod, Spherically-symmetric solutions of the Schrödinger-Newton equations, Class. Quantum Grav. 15 (1998) 2733-2742.

[9] L. D. Landau, E. M. Lifshitz (1980). Statistical Physics. Vol. 5 (3rd ed.). Butterworth-Heinemann.

[10] E. M. Lifshitz, L. P. Pitaevskii (1980). Statistical Physics, Part 2: Theory of the Condensed State. Vol. 9 (1st ed.). Butterworth-Heinemann.

[11] R. K. Dodd, J. C. Eilbeck, J. D. Gibbon, H. C. Morris, Solitons and Nonlinear Wave Equations (1982), Academic Press Inc., (London).

[12] W. A. Strauss (1978) Nonlinear Invariant Wave Equations, In Velo G and Wightman A (Ed.), Invariant Wave Equations, Berlin, Springer-Verlag.

[13] P. S. Lomdahl, O. H. Olsen and P. L. Christiansen (1980)
Return and Collapse of Solutions to the Nonlinear Schr dinger Equation in Cylindrical Symmetry, Phys. Lett., 78A, 125-128.

[14] K. Konno and H. Suzuki (1979) Self-focusing of Laser Beam in Nonlinear Media, Physica Scripta, 20, 382-386.

[15] V. E. Zakharov and V. S. Synakh (1975) The Nature of the Self-focusing Singularity, Zh. Eksp. Teor. Fiz. 68, 940-94.

[16] M. Rozner and V. Desjacques (2018) Backreaction of Axion Coherent Oscillations, arXiv: 1804.10417v1 [astro-ph.CO] 27 Apr 2018.

[17] J. Eby, M. Leembruggen, L. Street, P. Suranyi, and L. C. R. Wijewardhana (2019) Global View of QCD Axion Stars, arXiv: 1905.00981v3 [hep-ph] 9 Sep 2019.

[18] J. R. Lonnborn, A. Melatos, and B. Haskell (2019) Collective, Glitch-like Vortex Motion in a Neutron Star with an Annular Pinning Barrier, arXiv: 1905.02877v1 [astro-ph.HE] 8 May 2019.

[19] T. Harko (2019) Jeans Instability and Turbulent Gravitational Collapse of Bose-Einstein Condensate Dark Matter Halos arXiv: 1909.05022v1 [gr-qc] 9 Sep 2019.

[20] S. Sarkar, C. Vaz and L. C. R. Wijewardhana (2018) Gravitationally Bound Bose Condensates with Rotation, arXiv: 1711.01219v2 [astro-ph. GA] 15 May 2018. 